\title{
Targeted-Plasticity in the Corticospinal Tract After Human Spinal Cord Injury
}

\author{
Lasse Christiansen ${ }^{1,2} \cdot$ Monica A. Perez ${ }^{1,2}$ \\ Published online: 26 June 2018 \\ (C) The American Society for Experimental NeuroTherapeutics, Inc. 2018
}

\begin{abstract}
Spinal cord injury (SCI) often results in impaired or absent sensorimotor function below the level of the lesion. Recent electrophysiological studies in humans with chronic incomplete SCI demonstrate that voluntary motor output can be to some extent potentiated by noninvasive stimulation that targets the corticospinal tract. We discuss emerging approaches that use transcranial magnetic stimulation (TMS) over the primary motor cortex and electrical stimulation over a peripheral nerve as tools to induce plasticity in residual corticospinal projections. A single TMS pulse over the primary motor cortex has been paired with peripheral nerve electrical stimulation at precise interstimulus intervals to reinforce corticospinal synaptic transmission using principles of spike-timing dependent plasticity. Pairs of TMS pulses have also been used at interstimulus intervals that mimic the periodicity of descending indirect (I) waves volleys in the corticospinal tract. This data, along with information about the extent of the injury, provides a new framework for exploring the contribution of the corticospinal tract to recovery of function following SCI.
\end{abstract}

Keywords Noninvasive brain stimulation · Physiology of magnetic stimulation · Spinal cord injury $\cdot$ Rehabilitation $\cdot$ Spinal plasticity.

\section{Introduction}

Spinal cord injury (SCI) affects $\sim 17,000$ individuals per year in the USA. These individuals have limited motor function resulting in serious disability. During the past decades, experimental strategies - from neuroprotection to cell transplantation - have aimed at restoring sensorimotor function following SCI. Yet, these efforts have not resulted in effective treatments, and currently, neuromodulation with and without rehabilitation are some of the most promising approaches to achieve this goal. Motor recovery after SCI likely depends on the involvement of multiple descending motor pathways, including the corticospinal tract. In this review, we will address questions such as the extent to which corticospinal connections are still present after the injury and how can we boost their level of excitability. We first discuss anatomical,

Monica A. Perez

perezmo@miami.edu

1 Department of Neurological Surgery, The Miami Project to Cure Paralysis, University of Miami, Miami, FL 33136, USA

2 Bruce W. Carter Department of Veterans Affairs Medical Center, 1201 NW 16th Street, Miami, FL 33125, USA electrophysiological, and imaging data showing that most injuries to the spinal cord in humans are discomplete, indicating that there is some continuity of CNS tissue across the injured segments. Then, we discuss recent electrophysiological studies in humans with chronic incomplete SCI showing that voluntary motor output can be potentiated to some extent by approaches that use transcranial magnetic stimulation (TMS) and peripheral nerve stimulation as tools to induce plasticity of residual corticospinal projections.

\section{Spinal Cord Injuries Are Rarely Anatomically Complete}

The extent of SCI in humans is defined clinically by the American Spinal Injury Association Impairment Scale (AIS). Here, individuals with AIS A have no sensory or motor function preserved in sacral segments S4-S5 and they are classified as "clinically complete SCI". Individuals with AIS B have preserved sensory, but not motor, function below the neurologic level through sacral segments S4-S5 and individuals with AIS C and D, have different degrees of motor function preserved below the neurologic level and all of them are 
classified as "clinically incomplete SCI". Individuals with AIS E have normal sensory and motor function. The lack of voluntary motor output in humans with clinically complete SCI does not necessarily imply that spinal motoneurons caudal to the lesion are entirely deprived of descending innervation. Early postmortem analysis of spinal cords showed that $\sim 75 \%$ of individuals with a diagnosis of clinically complete SCI showed evidence of some continuity of CNS tissue across the injured segments [41]. Histological analysis of spinal cord lesions at their epicenter also revealed evidence of continuity of CNS parenchyma in $\sim 62 \%$ of specimens [7]. In agreement, earlier neurophysiological studies showed that individuals with clinically complete SCI could present a tonic vibratory response [21,22], voluntarily suppress responses to stimulation [11], and respond to reinforcement maneuvers [21,22], suggesting that some supraspinal control in muscles below the level of the injury was preserved. A combination of these and other methods indicated that a rather large proportion of humans with clinically complete SCI showed one or more signs of spared connections [57]. Therefore, these individuals were categorized as discomplete [20].

Contemporary evidence continues to support the view that a large number of individuals with clinically complete SCI are discomplete. Recently, it was found that $\sim 66 \%$ of individuals with a diagnosis of AIS A and B were able to produce volitional electromyographic signals in muscles which motoneurons were located below their injury level [37]. Most individuals with clinically complete SCI had responses evoked by TMS over the primary motor cortex and/or voluntary muscle activity in muscles innervated below the lesion $[26,59]$. Vestibular-evoked myogenic potentials have been observed in a small number of participants, suggesting preservation of other descending motor pathways [59]. Behavioral evidence of the discomplete condition also comes from studies using epidural or transcutaneous spinal cord stimulation combined with motor training showing recovery of some function in individuals with clinically complete SCI $[2,23$, $36,62]$. It is important to note that from the first to the fifth year following a SCI, $\sim 2.1 \%$ of individuals progress from motor complete (AIS A and B) to incomplete (AIS C and D; [44]). This is in contrast with the rather large fraction of discomplete patients who theoretically could develop meaningful motor behaviors. Several factors could contribute to these findings, including the lack of sensitivity of current clinical assessments to examine changes in sensorimotor outcomes over time [48] and the intensity used in training regimes, which does not allow people with clinically complete SCI to have any recovery in muscles below the level of the injury [30]. Overall, it is evident that residual connections are present in a large number of humans after SCI and we discuss how increasing transmission in those connections might open avenues for motor recovery.

\section{How Do We Activate Residual Corticospinal Projections After SCl?}

Magnetic stimulation of the scalp provides an indirect mean of inducing electrical current over the primary motor cortex to generate motor evoked potentials (MEPs) in different muscle groups. This is achieved by using a short-lasting magnetic field that peaks after $0.2 \mathrm{~ms}$ and readily penetrates to the cortex due to the low impedance of the scalp. Since the magnetic field does not activate nociceptors this is done with minimal discomfort as compared to electrical stimulation of the scalp. The short-lasting field of most commercially available stimulators favors excitation of axons over cell bodies and a rapid decline in intensity at distance from superficial cortical layers. Cortical neurons are most likely to be activated where a change of voltage along the axon occurs; this is where an axon bends away from the direction of the magnetic field [1, 47].

A single TMS pulse over the primary motor cortex evokes temporally synchronized descending waves in the corticospinal tract that can be recorded from the epidural space $[18,54]$. The earliest wave is likely due to direct stimulation of the corticospinal neuron (D-wave) at some distance from the cell body, while the later indirect (I) waves (termed I1, I2, and I3) possible arise from transsynaptic activation of corticospinal neurons by intracortical circuits [18]. Evidence suggested that changes in the latency of MEPs elicited by different TMS-induced currents in the brain can be used to make inferences about different excitatory inputs to corticospinal neurons [18, 19]. For example, TMS-induced electrical currents flowing from posterior to anterior (PA) across the central sulcus preferentially evoke highly synchronized corticospinal activity, while currents flowing from anterior to posterior (AP) preferentially evoke less synchronized activity with their peaks partially matching the timing of the PA-evoked activity $[16,56]$. The characteristics of PA and AP activity resemble I-waves recorded in animal studies [43, 54] and the interval between I-waves in primates [49] and humans [17] is similar. MEPs elicited by PA and AP currents also change to different extents during voluntary motor tasks, motor learning, and plasticity protocols [18, 28, 33, 34, 46], suggesting that the assessment of these responses might provide insights into the understanding of behaviors and modulation of central motor circuits. Notably duration and intensity of the field as well as the direction of the induced current in the brain affects the characteristics of MEPs elicited by TMS in control subjects $[25,58]$ and in humans with SCI [40]. In control subjects, MEPs elicited with the coil in the AP orientation have longer latency, larger latency dispersion, and higher threshold than MEPs elicited in the PA orientation $[18,19]$. Orienting the coil to induce currents flowing from lateral to medial (LM) favors direct activation of the corticospinal neurons resulting in MEPs with shorter latencies compared with PA and AP stimulation [70]. Recent evidence 
showed that MEP latencies in all coil orientations are prolonged in humans with SCI compared with control subjects [40]. In addition, latencies of MEPs elicited by PA and AP stimulation relative to those elicited by LM stimulation are shorter in SCI compared with control subjects and in particular for AP MEPs, suggesting that neural structures activated by AP induced currents are largely affected after SCI [40]. Although insights have been gained about how to stimulate residual corticospinal tract connection following SCI, effective protocols that engage these connections to facilitate motor recovery still remain limited $[32,65]$. We discuss here two emerging protocols that target the corticospinal tract in humans with SCI, one of them based on the principles of spike-timing dependent plasticity (STDP) and the other based on the frequency of repetitive I-wave descending volleys. We argue that targeted TMS protocols can engage corticospinal plasticity and thereby by a useful tool to enhance motor function after SCI.

\section{Stimulation Based on the Principles of STDP}

STDP refers to a process by which synaptic strength changes after correlational pre- and postsynaptic spiking [3]. The temporal characteristics of the critical window vary between neural tissues. In pyramidal layer 5 cells e.g. the critical window resembles that of hippocampal culture [50]. When presynaptic activation precedes postsynaptic antidromic somatic activation, synaptic connectivity increases whereas the reversed order results in a decline in synaptic strength. As suggested by Hebb [38], the temporal characteristics call for a causality detection mechanisms rather than mere coincidence recognition. The $N$-methyl-D-aspartate (NMDA) glutamate receptor can serve this purpose [42]. Both in vitro STDP [8] and STDPlike changes in humans [24] are thought to engage long-term potentiation (LTP) and long-term depression (LTD) mechanisms that depend on NMDA receptor activity. Timely arrival of the antidromic postsynaptic activation removes the $\mathrm{Mg} 2+$ block which enables $\mathrm{Ca} 2+$ to diffuse through the NMDA receptor [42]. The NMDA receptor thusly detects the postsynaptic depolarization and the presynaptic release of glutamate. The resultant change in Ca2+ influx leads to LTD or LTP induction dependent on the intracellular calcium concentration.

Noninvasive-paired associative stimulation protocols using principles of STDP were first used in humans to target the primary motor cortex [60]. Evidence showed that afferent volleys timed to arrive at the somatosensory cortex at the time of magnetic discharge led to an increase in corticospinal excitability [61]. However, if afferent volleys arrived to the primary motor cortex $\sim 15 \mathrm{~ms}$ before the magnetic stimulation (i.e., interstimulus interval between paired pulses of $10 \mathrm{~ms}$ ), inhibition replaced corticospinal facilitation [71]. Accordingly, the underlying mechanisms were thought to be changes in synaptic efficacy due to LTP- and LTD-like processes. Since the observed effects arise from changes in populations of neurons on a much larger scale than the cellular STDP, this noninvasive form of plasticity is often referred to as STDP-like changes. In humans with SCI, protocols resulting in STDP-like changes have targeted the sensorimotor cortex [55] and the spinal cord $[5,6,68]$. One of the possible limitations of STDP-like protocols targeting the sensorimotor cortex after SCI is the susceptibility to impaired ascending transmission. In addition, little is known about the effect of STDP-like protocols targeting the sensorimotor cortex on motor function. Consequently targeting the corticospinal tract caudal to lesion potentially entails a greater clinical utility. In the next section, we therefore restrict the discussion to recent findings using STDP-like protocols targeting the spinal cord.

STDP-like changes in the spinal cord were first demonstrated by Taylor and Martin [64] in control subjects. The authors showed that when repeated corticospinal activity triggered by TMS over a region of the primary motor cortex controlling the biceps brachii was timed to arrive a few milliseconds before antidromic activation of the motoneurons caused by supramaximal peripheral nerve electrical stimulation, the size of cervicomedullary MEPs (CMEPs) increased. In contrast, when postsynaptic depolarization preceded the presynaptic activation, the size of CMEPs decreased. CMEPs measure corticospinal transmission and/ or excitability of spinal motoneurons [63], suggesting that the results indicated the presence of plasticity at the spinal cord. Since this plasticity targeted synapses between corticospinal neurons and spinal motoneurons using pairs of stimuli, it has been referred to as to paired corticomotoneuronal stimulation (PCMS). Note that the precise timing of the two stimuli is based on measurements of central and peripheral motor conduction time. Conduction times are estimated from the latency of a MEP, the latency of a response elicited by cervical root stimulation along with latency of the M-wave and F-wave. Bunday and Perez [5] reproduced those results in control subjects and for the first time used PCMS in humans with incomplete SCI (Fig. 1). In their study, the size of MEPs elicited by electrical and magnetic stimulation of the primary motor cortex and CMEPs increased and decreased according to the timing of arrival of volleys in the pre- and postsynaptic terminals without changes in F-waves [5]. Although this evidence suggests changes at corticospinal-motoneuronal synapses as a likely mechanism, these results need to be interpreted with caution. Limitations have been described in the extent to which F-wave measurements can assess motoneuron excitability $[27,39]$.

PCMS has also been used to target lower limb muscles following SCI [68]. In agreement with the results found in arm [29] and hand [5, 6] muscles, testing in the leg showed 

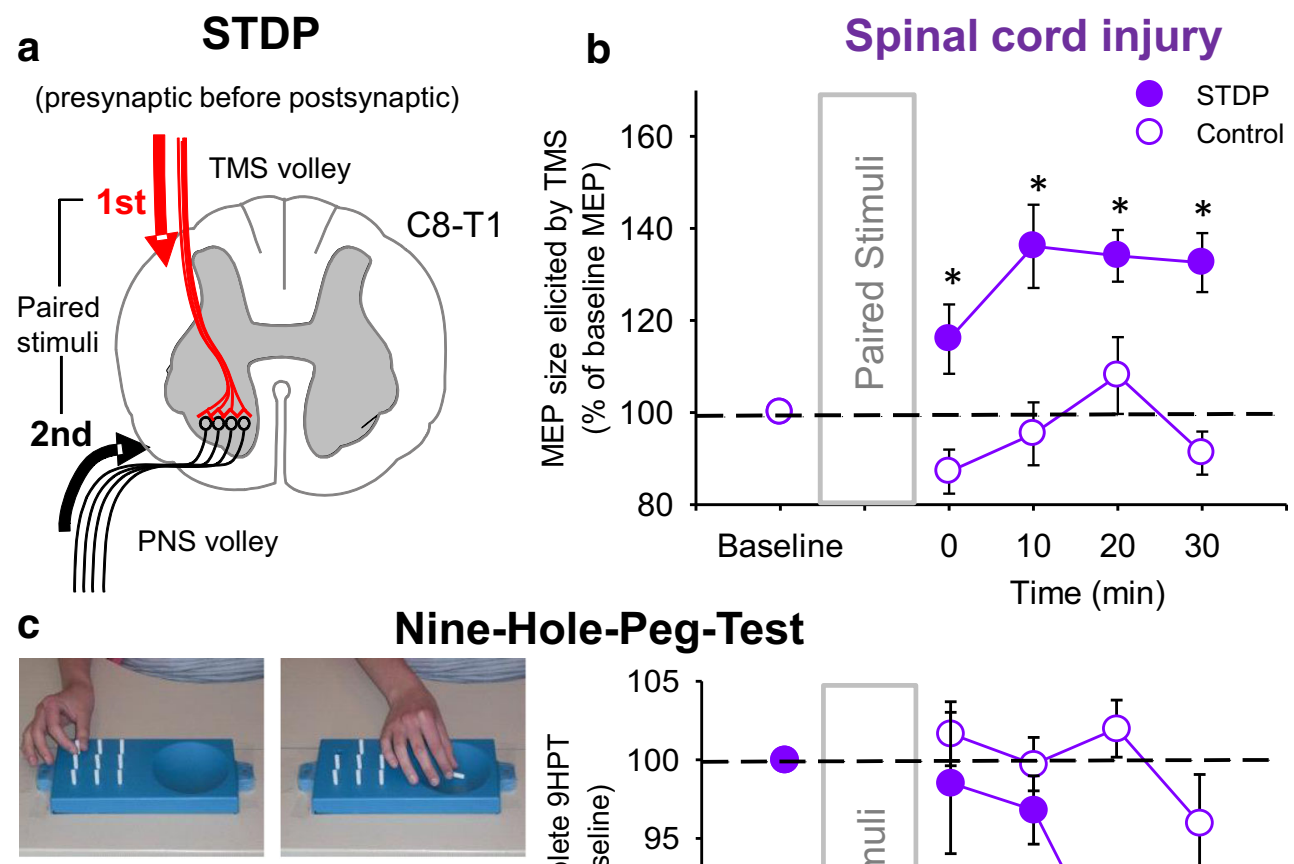

Nine-Hole-Peg-Test
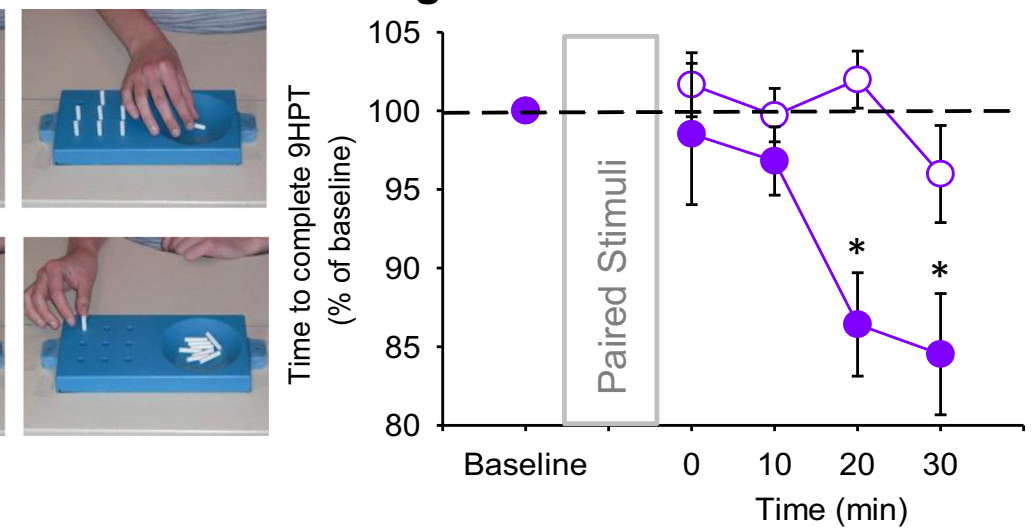

Fig. 1 (a) Illustration of the paired corticomotoneuronal stimulation (PCMS) protocol used to enhance corticospinal function after SCI. Here, corticospinal neurons were activated at a cortical level by using transcranial magnetic stimulation (TMS volley, 1st) delivered over the hand primary motor cortex and spinal motoneurons were activated antidromically by peripheral nerve stimulation (PNS volley, 2nd)

delivered to the ulnar nerve. (b) Motor evoked potentials (MEPs) size and hand function tested by the 9-hold-peg test increased after PCMS but not a control protocol (where postsynaptic pulses were timed to arrive to the synapses $5 \mathrm{~ms}$ before presynaptic activation) (c) in 18 participants with chronic cervical SCI. Error bars, SEs, $* p<0.05$. Modified from [5]

that increasing the number of pairs of stimuli resulted in larger increases in corticospinal excitability (Perez lab, unpublished observation), supporting the view that PCMS effects are dosedependent. In the lower limb, the excitatory effects of PCMS were similar to those found in upper limb muscles but not the inhibitory effects. The inhibitory effects of PCMS in the tibialis anterior muscle were contingent on a suppressive effect of peripheral nerve stimulation on the MEP present during the conditioning protocol [68]. The neurophysiological mechanisms underlying this interindividual variability are yet to be determined, but the supramaximal nature of the peripheral stimulation suggest that likely both homonymous and heteronomous mixed nerve activation contributed to the effects leading to a complex integration at a spinal level. Aside from PCMS protocols, other paradigms have been used to induce plasticity at the spinal level [14, 45]. These studies showed that repeated electrical stimulation of the posterior tibial nerve with TMS over the leg motor cortex at different stimulus intervals increased the size of the soleus H-reflex. However, in these protocols, the time of arrival of volleys at the presynaptic or postsynaptic was not quantified nor was the ability to suppress the size of responses, which is critical to make inferences about STDP-like plasticity.

At a behavioral level, evidence showed that PCMS increases voluntary activation of low threshold motor units as evidenced by increases in low levels of electromyography (EMG) and force generation [5, 64, 68] and to a lesser extent increases the level of maximal voluntary contraction [15]. In humans with SCI, changes in corticospinal transmission positively correlated with enhancements in voluntary motor output, suggesting an association between motor output and the strength of the induced plasticity [5]. In addition, the clinical potential was highlighted by decreases in the time to complete the 9-hole peg test reflecting improvements in fine motor function. Recently, these findings were extended by showing that the effect of PCMS on corticospinal excitability could be augmented when the pairs of stimuli 
were delivered during short-lasting low-intensity isometric voluntary contractions [6]. Increasing the excitability of the motoneurons through voluntary activation augmented the increases in the size of MEPs and reversed nonresponders to responders. Since spinal lesions are associated with reduced corticospinal inputs to the motoneurons, the possibility for potentiating PCMS aftereffects with voluntary activation increases the clinical potential substantially. Changes in the number and size of descending volleys elicited by voluntary contraction might contribute to the larger increases in MEPs size elicited in SCI participants. The expected outcome would be an increased corticospinal facilitation, which was evident as an increased number of responders and as increased facilitation for individuals with SCI who responded to the PCMS at rest (Fig. 2). In addition, evidence showed a similar augmentation in corticospinal excitability when PCMS was administered in combination with acute intermittent hypoxia, which is a noninvasive strategy known to increase corticospinal plasticity [10]. Taken together, these findings suggest that PCMS alone or in combination with other noninvasive therapeutic protocols can enhance the beneficial effects of sensorimotor rehabilitation after SCI.

a

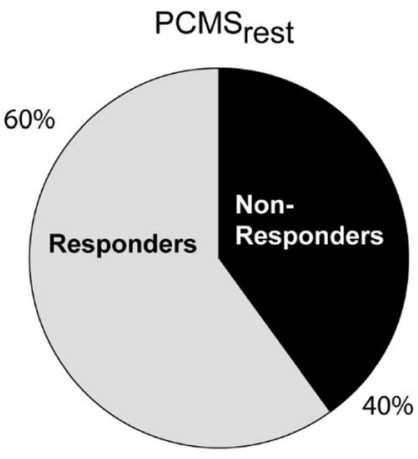

b

Non-Responders

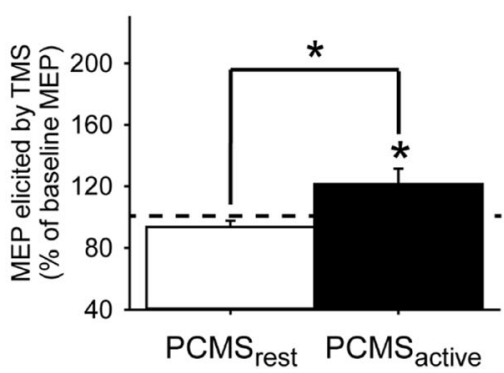

Fig. 2 MEPs after PCMS in responders and nonresponders. SCI participants were grouped into responders and non-responders based on the amount of TMS MEP facilitation after PCMS applied at rest $\left(\mathrm{PCMS}_{\text {rest }}\right)$ and during voluntary activity $\left(\mathrm{PCMS}_{\text {active }}\right)$. (a, c) Pie charts show the proportion of responders $(n=9)$ and non-responders $(n=6)$. (b, d) Graphs show the subgroup data. The abscissa shows the paired-pulse protocol and the ordinate shows the average (across 0 and $30 \mathrm{~min}$ ) first

\section{Stimulation Based on the Frequency of I-Waves}

The summation of D- and I-waves at the motoneuronal pool likely contributes to the generation of MEPs [18]. The temporal organization of corticospinal volleys necessary to bring motoneurons to threshold is impaired following SCI [4], suggesting that understanding transmission of descending volleys is relevant for the generation of motor output after the injury [13]. Studies using pairs of TMS have shown that it is possible to make inferences about the physiology of I-waves from surface EMG recordings. Paired-TMS pulses can be precisely timed to increase the amplitude of MEPs at interstimulus intervals of $\sim 1.5 \mathrm{~ms}$ compatible with the I-waves recorded from the epidural space in control subjects $[67,72]$ referred as to short-interval intracortical facilitation (SICF). The principle here is that the 2nd pulse (subthreshold) indirectly excites the pyramidal neurons at a time of increased firing probability due to the excitatory postsynaptic potentials (EPSPs) triggered by the first supra-threshold stimulus. During SICF measurements, three distinct MEP peaks are observed at $\sim 1.5,3$ and $4.5 \mathrm{~ms}$ [12, 67, 72], which are compatible with the peaks of the I-waves recorded from the epidural space. Evidence
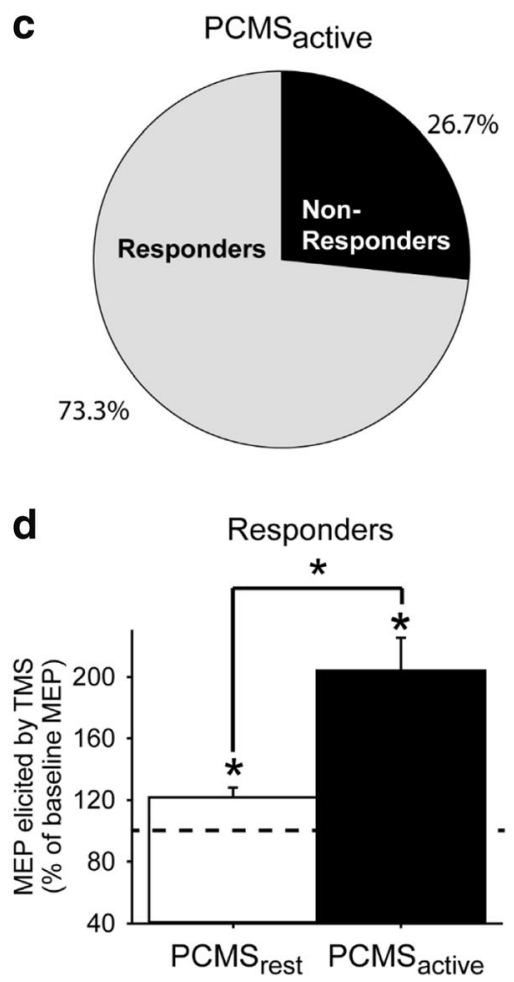

dorsal interosseous (FDI) MEP size in non-responders (b; open bar: $\mathrm{PCMS}_{\text {rest }}$; closed bar: $\mathrm{PCMS}_{\text {active }}$ ) and in responders (d; open bar: $\mathrm{PCMS}_{\text {rest }}$; closed bar: $\mathrm{PCMS}_{\text {active }}$ ) as a \% of baseline MEP. Note that in responders, the size of MEPs increased after PCMS applied at rest and further increased after PCMS applied during voluntary activity whereas in non-responders MEP size increased only after PCMS ${ }_{\text {active. }}$ Error bars indicate the SE $* p<0.05$. Modified from Bunday et al. 2018 
showed that the first MEP peak has a cortical origin $[67,72]$ while the second and third MEP peak likely reflect contributions from cortical and subcortical pathways [12]. Notably, a study showed a decreased in the amplitude of all three MEP peaks and a delayed and an increased duration of the third MEP peak in humans with SCI compared with control subjects [13]. A mathematical model used to estimate the relative contribution from the different MEP peaks to the recruitment of motor units showed that the third MEP peak contributed mainly to the recruitment of high-threshold motor units in control subjects $[13,66]$. In contrast, the third MEP peakwhich is the one more affected by SCI - aberrantly contributed to the recruitment of all types of motor units [13]. This is in line with results showing impaired motoneuronal capacity to summate EPSPs coming from Ia afferents following SCI [52]. Other studies also suggested that neural structures contributing to the generation of late I-wave and/or the third MEP peak measured by SICF are affected following SCI. For example, MEPs elicited by AP compared with PA stimulation were shorter in SCI compared with control subjects [40]. MEPs elicited by AP stimulation likely involve contributions from oligosynaptic circuits from multiple brain areas [31, 69], which might be difficult to recruit after SCI because of changes in functional connectivity between cortical regions after the injury [51]. In addition, MEPs elicited by AP stimulation are modulated by afferent inhibition to a larger extent than MEPs elicited by PA stimulation [35] and afferent input is altered after SCI [53]. All together, these results indicate that neural structures contributing to the generation of late I-wave volleys are affected following SCI; thus, these circuits might represent a putative target for noninvasive plasticity protocols aiming at promoting motor function.

In agreement, Long, Federico et al. [46] demonstrated for the first time that pairs of TMS pulses at an interstimulus interval mimicking the late I-wave volleys increased corticospinal excitability and fine motor function in humans with incomplete cervical SCI. The study showed in participants with chronic incomplete SCI (AIS C and D), increases in voluntary motor output and decreases in the time to complete the 9-hole-peg test after 180 pairs of pulses. These changes were observed after the protocol that used a late Iwave interstimulus interval but not after a control protocol with an interstimulus interval in between the I-wave peaks. In the study, several of the results supported the view that during the stimulation, it was possible to target volleys with characteristics resembling late I-waves on corticospinal neurons. First, electrophysiological assessments revealed an increase in amplitude of MEPs elicited by AP but not PA stimulation indicating that changes in corticospinal transmission were specific to the neural structures activated by AP currents. Second, it was found that SICF, testing the third MEP peak, increased after the I-wave stimulation protocol. SICF at the interstimulus interval measured in the study likely reflects summation of late I-wave inputs $[18,56]$. It was also shown that the activity in spinal cord circuits contributed to the results since it was found that the amplitude and persistence of the Fwaves increased after the I-wave protocol in both groups of subjects. An important question is, how repeated activation of neural structures contributing to the generation of late I-waves changed spinal cord excitability. A possibility is that better synchronized and/or larger corticospinal volleys traveling to spinal motoneurons were evoked by the I-wave stimulation protocol and contributed to this result. This is supported by the decreased onset latency dispersion for MEPs tested by AP but not by PA stimulation. Reaction times are prolonged in people with SCI compared with uninjured individuals [28] and prolonged reaction times in SCI subjects have been related to changes in the third MEP peak during SICF testing [13]. This might to some extent reflect the longer time needed to raise spinal motoneurons to threshold because of the lesser descending drive after the injury. Evidence showed that motoneurons of subjects with SCI are activated by longer periods of depolarization compared with uninjured subjects [52]. The latency of EPSPs on motoneurons shows a relation with the latencies of I-waves [43]. Therefore, it is possible that the lesser variability on the latency of MEPs elicited by AP stimulation after the I-wave protocol reflected larger and/or better synchronized corticospinal descending volleys, which might be translated to changes in motor output and movement speed. This is supported by the decreased time needed to complete a dexterity test and the more EMG and force outcomes exerted during ballistic index finger voluntary contractions after the Iwave protocol (Fig. 3).

\section{Conclusions and Future Directions}

Since spinal cord injuries are rarely complete, the possibility of accessing remaining spared corticospinal projections to induce functional recovery is not as low as one might think. Both the PCMS and I-wave protocols targeted physiological connections involving the corticospinal tract weakened by SCI. The PCMS protocol aims to strengthen the connection between corticospinal neurons and motoneurons. By targeting excitatory inputs to corticospinal neurons, the I-wave protocol induced plasticity at both cortical and spinal levels resulting in improved synchronization of corticospinal responses and motor output. Note that both protocols based their design on electrophysiological information about motor control mechanisms, suggesting that electrophysiology can be successfully used to guide interventions aiming to enhance motor output in humans with SCI.

Several aspects need to be considered for future translation of these protocols to a clinical environment. First, it will be important to assess the effectiveness of PCMS using lower intensities since current PMCS protocols in humans with 

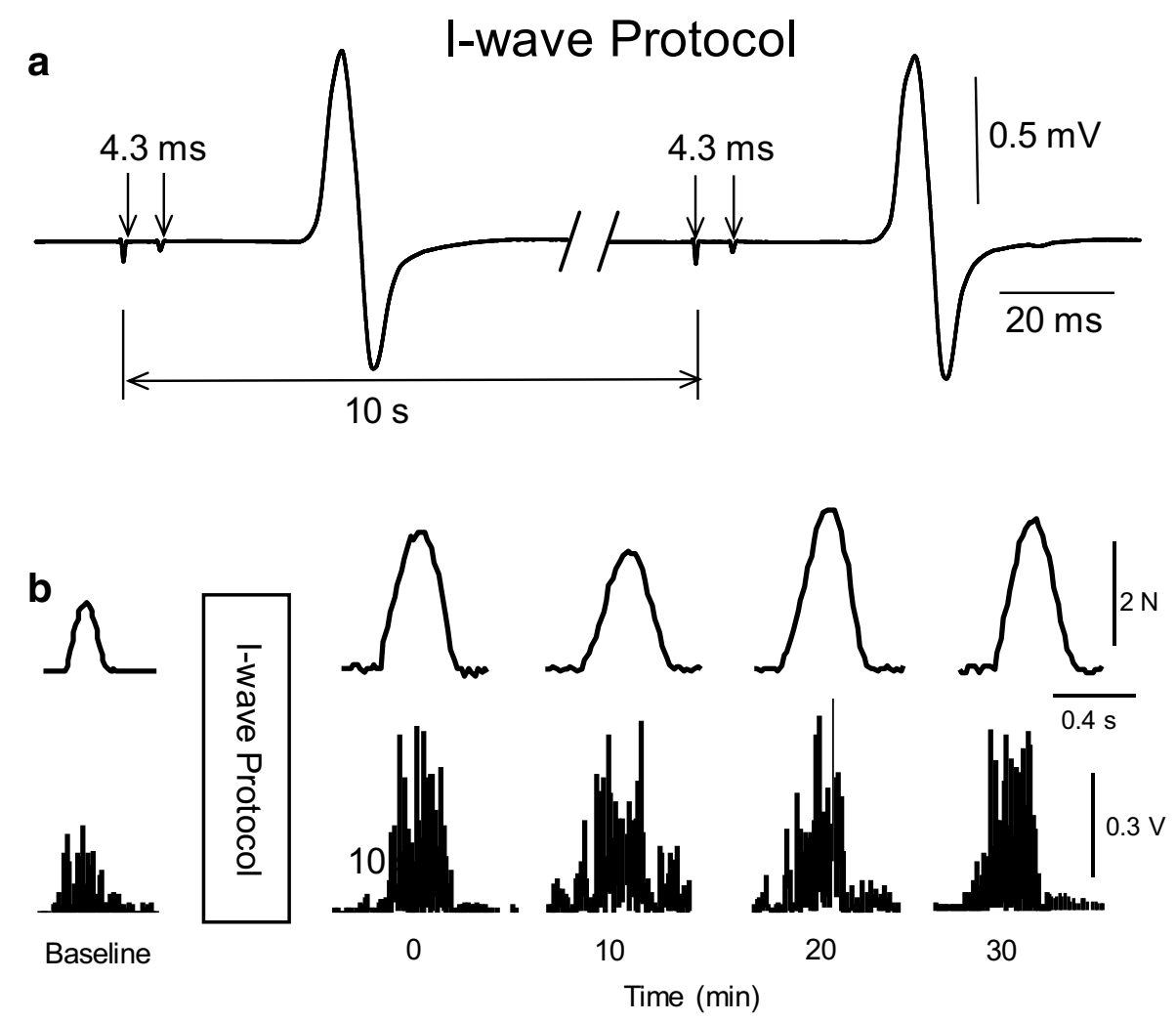

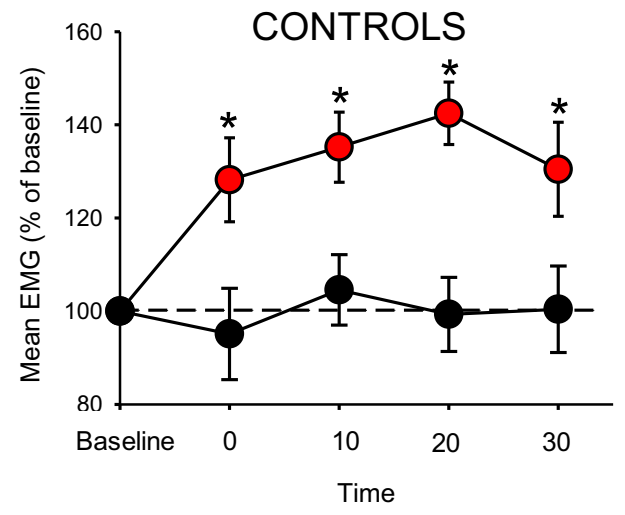

Fig. 3 Experimental setup. (a) Conditioned MEPs recorded from a representative subject by using pairs of TMS pulses delivered at an interstimulus interval of $4.3 \mathrm{~ms}$ (I-wave protocol) and $3.5 \mathrm{~ms}$ (control protocol; not shown). Note that in both protocols, the intertrial interval was $10 \mathrm{~s}$. (b) Voluntary motor output was assessed by examining changes in mean force and mean rectified electromyography (EMG) activity during brief, fast, index finger voluntary contractions in the abduction

SCI use $100 \%$ of maximal stimulator output (MSO). In control subjects, submaximal stimulation intensities $(\sim 70 \%$ of MSO) have shown to be successful in inducing both physiological and behavioral plasticity, suggesting that lower intensities might have beneficial effects. The same principle applies for the I-wave protocol. Higher motor thresholds in humans with SCI might force the use of higher TMS intensities to elicit MEPs in selected muscles. Indeed, close to maximal intensity is often needed to excite motoneurons to the plateau level or

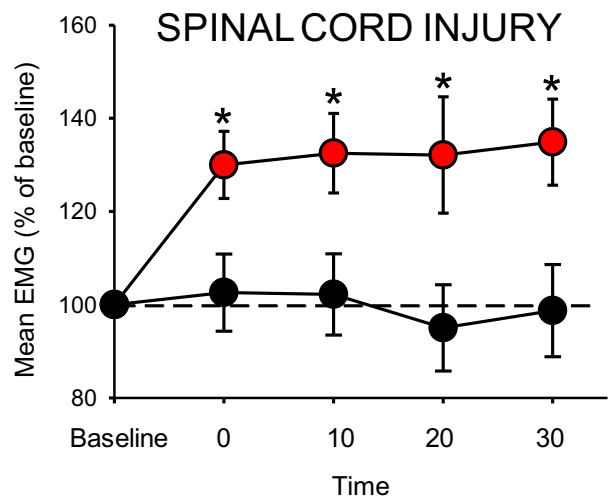

direction before (baseline) and after $(0,10,20$, and $30 \mathrm{~min})$ the I-wave protocol. Raw force (upper) and EMG (lower) traces from a representative SCI subject. Group data show changes in mean rectified EMG activity in controls (left side graph) and SCI participants (right side graph) after the I-wave (red circles) and control (black circles) protocol. Error bars indicate the SE. $* p<0.05$. Modified from Long et al. [46]

even just above firing threshold after SCI. However, the high intensity stimulation used in SCI participants may serve a dual purpose. A high intensity ensures adequate activation of corticospinal neurons damaged by the injury. In agreement, combining PCMS with voluntary contraction of the target muscle when possible ensures adequate excitation of motoneurons to favor successful outcomes. A main concern for the clinical use of the PMCS protocol is the need for electrophysiological finesse when determining the interstimulus intervals 
needed between TMS and peripheral nerve electrical stimulation in individual subjects. It can be argued that this reduces its clinical applicability. A possible solution is to apply a train of a high-frequency peripheral nerve stimulation, which could increase the temporal window leading to facilitation, with the drawback that this less likely relies on spike-timing dependent mechanisms. On the other side, electrophysiological times needed for PMCS are outcomes obtained in clinical diagnostic procedures [9]. In addition, latencies of EMG responses, which depend on the generation of action potentials to the muscle, are used for PMCS estimations. Thus, proper training and careful methodological considerations can represent the next step for bringing this strategies to the clinic. One might also envision that specialized neurophysiologists might be needed for determining the precise timing and that after that determination the protocol can be readily applied for massive use in the clinic. Similar concern arise for the I-wave protocol, since ideally the interstimulus interval used by pairs of TMS pulses might better rely on the presence of facilitation at the third MEP peak. Note that here there is not one but several interstimulus intervals that might result in facilitation in this protocol and therefore a proper time course for determining the interval might add sensitivity to the intervention. It is difficult to compare the I-wave protocol to other repetitive noninvasive brain stimulation paradigms resulting in plasticity since here pairs of pulses are given every several seconds with resting periods in between. The interval between pairs of TMS stimuli used in the I-wave protocol is shorter than what has been used in theta burst stimulation in humans and longer than what has been used for sensorimotor cortex stimulation in rodents. Overall, both approaches represent new promising tools but further investigations into underlying mechanisms, optimal dose, size, and duration of effects along with applicability out of laboratory settings are yet to be adequately determined. The effects also need to be examined in multicenter clinical trials where the potential for cumulative effects of several sessions along with interactions with motor practice could be explored, which represents the next step in translational medicine.

Required Author Forms Disclosure forms provided by the authors are available with the online version of this article.

\section{References}

1. Amassian VE, Eberle L, Maccabee PJ, Cracco RQ. Modelling magnetic coil excitation of human cerebral cortex with a peripheral nerve immersed in a brain-shaped volume conductor: the significance of fiber bending in excitation. Electroencephalogr Clin Neurophysiol 1992; 85:291-301.

2. Angeli CA, Edgerton VR, Gerasimenko YP, Harkema SJ. Altering spinal cord excitability enables voluntary movements after chronic complete paralysis in humans. Brain 2014; 137: 1394-1409.
3. Bi Gq. and Poo Mm. Synaptic modifications in cultured hippocampal neurons: dependence on spike timing, synaptic strength, and postsynaptic cell type. J Neurosci 1998; 18: 10464-10472.

4. Brouwer P, Bugaresti J, Ashby P. Changes in corticospinal facilitation of lower limb spinal motor neurons after spinal cord lesions. $\mathrm{J}$ Neurol Neurosurg Psychiatry 1992; 55(1): 20-24

5. Bunday KL and Perez MA. Motor recovery after spinal cord injury enhanced by strengthening corticospinal synaptic transmission. Curr Biol 2012; 22: 2355-2361.

6. Bunday KL, Urbin MA, Perez MA. Potentiating paired corticospinal-motoneuronal plasticity after spinal cord injury. Brain Stimul (2018). https://doi.org/10.1016/j.brs.2018.05.006.

7. Bunge RP, Puckett W, Becerra J, Marcillo A, Quencer R. Observations on the pathology of human spinal cord injury. A review and classification of 22 new cases with details from a case of chronic cord compression with extensive focal demyelination. Adv Neurol 1993 59: 75.

8. Caporale N, Dan Y. Spike timing-dependent plasticity: a Hebbian learning rule. Annu Rev Neurosci 2008; 31: 25-46.

9. Chen R, Cros D, Curra A, et al. The clinical diagnostic utility of transcranial magnetic stimulation: report of an IFCN committee. Clin Neurophysiol. 2008;119: 504-532.

10. Christiansen L, Urbin MA, Mitchell GS, Perez MA. Acute intermittent hypoxia enhances corticospinal synaptic plasticity in humans. eLife 2018; 7: e34304.

11. Cioni B, Dimitrijevic MR, McKay WB, Sherwood AM. Voluntary supraspinal suppression of spinal reflex activity in paralyzed muscles of spinal cord injury patients. Exp Neurol 1986; 93: 574-583.

12. Cirillo J, Perez MA. Subcortical contribution to late TMS-induced I-waves in intact humans. Front Integr Neurosci 2016 9: 38.

13. Cirillo JF, Calabro L, Perez MA. Impaired organization of pairedpulse TMS-induced I-waves after human spinal cord injury. Cereb Cortex 2015; 26: 2167-2177.

14. Cortes M, Thickbroom GW, Valls-Sole J, Pascual-Leone A, Edwards DJ. Spinal associative stimulation: a non-invasive stimulation paradigm to modulate spinal excitability. Clin Neurophysiol 2011; 122(11): 2254-2259.

15. D'Amico JM, Donges SC, Taylor JL. Paired corticospinalmotoneuronal stimulation increases maximal voluntary activation of human adductor pollicis. J Neurophysiol 2017: jn-00919.

16. Day BL, Dressler D, Maertens de Noordhout A, Marsden CD, Nakashima K, Rothwell JC, Thompson PD. Electric and magnetic stimulation of human motor cortex: surface EMG and single motor unit responses. J Physiol 1989; 412: 449-73. Erratum in: J Physiol (Lond) 1990 Nov; 430: 617.

17. Di Lazzaro V, Oliviero A, Profice P, et al. Comparison of descending volleys evoked by transcranial magnetic and electric stimulation in conscious humans. Electroencephalogr Clin Neurophysiol 1998; 109: 397-401.

18. Di Lazzaro V, Profice P, Ranieri F, et al. I-wave origin and modulation. Brain Stimul 2012; 5: 512-525.

19. Di Lazzaro V, Rothwell JC, Capogna M. Noninvasive stimulation of the human brain: activation of multiple cortical circuits. Neuroscientist 2017. https://doi.org/10.1177/1073858417717660.

20. Dimitrijević MR. Residual motor functions in spinal cord injury. Advances in neurology 1988; 47:138-155

21. Dimitrijevic MR, Spencer WA, Trontelj JV, Dimitrijevic M. Reflex effects of vibration in patients with spinal cord lesions. Neurology 1977; 27: 1078-1078.

22. Dimitrijevic MR, Dimitrijevic MM, Faganel J, Sherwood AM. Suprasegmentally induced motor unit activity in paralyzed muscles of patients with established spinal cord injury. Ann Neurol 1984; 16: 216-221.

23. Donati ARC, Shokur S, Morya E, et al. Long-term training with a brain-machine interface-based gait protocol induces partial neurological recovery in paraplegic patients. Sci Rep 2016; 6: 30383. 
24. Donges SC, D'Amico JM, Butler JE, Taylor JL. The involvement of N-methyl-D-aspartate receptors in plasticity induced by paired corticospinal-motoneuronal stimulation in humans. J Neurophysiol 2017; jn. 00457.02017.

25. D'Ostilio K, Goetz SM, Hannah R, et al. Effect of coil orientation on strength-duration time constant and I-wave activation with controllable pulse parameter transcranial magnetic stimulation. Clin Neurophysiol 2016; 127: 675-683.

26. Edwards D, Cortes M, Thickbroom G, Rykman A, Pascual-Leone A, Volpe B. Preserved corticospinal conduction without voluntary movement after spinal cord injury. Spinal Cord 2013; 51: 765.

27. Espiritu MG, Lin CSY, Burke D. Motoneuron excitability and the F wave. Muscle Nerve 2003; 27: 720-727.

28. Federico P, Perez, MA. Distinct corticocortical contributions to human precision and power grip. Cereb Cortex 2016; 27.11: 50705082.

29. Fitzpatrick SC, Luu BL, Butler JE, Taylor JL. More conditioning stimuli enhance synaptic plasticity in the human spinal cord. Clin Neurophysiol 2016; 127: 724-731.

30. Gant KL, Nagle KG, Cowan RE, et al. Body system effects of a multi-modal training program targeting chronic, motor complete thoracic spinal cord injury. J Neurotrauma 2018; 35: 411-423.

31. Groppa S, Werner-Petroll N, Münchau A, Deuschl G, Ruschworth MF, Siebner HR. A novel dual-site transcranial magnetic stimulation paradigm to probe fast facilitatory inputs from ipsilateral dorsal premotor cortex to primary motor cortex. Neuroimage 2012; 62: 500-509.

32. Gunduz A, Rothwell JC, Vidal J, Kumru H. Non-invasive brain stimulation to promote motor and functional recovery following spinal cord injury. Neural Regen Res 2017; 12: 1933-1938.

33. Hamada M, Murase N, Hasan A, Balaratnam M \& Rothwell JC (2013). The role of interneuron networks in driving human motor cortex plasticity. J Neurosci (23):1593-1605.

34. Hamada M, Galea JM, Di Lazzaro V, Mazzone P, Ziemann U \& Rothwell JC. Two distinct interneuron circuits in human motor cortex are linked to different subsets of physiological and behavioral plasticity. J Neurosci 2014; (34):12837-12849.

35. Hannah R, Rothwell JC. Pulse duration as well as current direction determines the specificity of transcranial magnetic stimulation of motor cortex during contraction. Brain Stimul 2017; 10: 106-115.

36. Harkema S, Gerasimenko Y, Hodes J, et al. Effect of epidural stimulation of the lumbosacral spinal cord on voluntary movement, standing, and assisted stepping after motor complete paraplegia: a case study. The Lancet 2011; 377: 1938-1947.

37. Heald E, Hart R, Kilgore K, Peckham PH. Characterization of volitional electromyographic signals in the lower extremity after motor complete spinal cord injury. Neurorehabil Neural Repair 2017; 31: $583-591$

38. Hebb D. 1949. The organization of behavior. New York: Wiley.

39. Hultborn H, Nielsen JB H-reflexes and F-responses are not equally sensitive to changes in motoneuronal excitability. Muscle Nerve 1995; 18: 1471-1474.

40. Jo HJ, Di Lazzaro V, Perez MA. Effect of coil orientation on motor evoked potentials in humans with tetraplegia. J Physiol 2018; in press.

41. Kakulas A. The applied neurobiology of human spinal cord injury: a review. Spinal Cord 1988 26: 371.

42. Kampa BM, Clements J, Jonas P, Stuart GJ. Kinetics of Mg2+ unblock of NMDA receptors: implications for spike-timing dependent synaptic plasticity. J Physiol 2004; 556: 337-345.

43. Kernell D, Chien-Ping W. Responses of the pyramidal tract to stimulation of the baboon's motor cortex. J Physiol 1967; 191: 653-672.

44. Kirshblum S, Millis S, McKinley W, Tulsky D. Late neurologic recovery after traumatic spinal cord injury. Arch Phys Med Rehabil 2004; 85: 1811-1817.
45. LeukelC, Taube W, Beck S, Schubert M. Pathway-specific plasticity in the human spinal cord. Eur J Neurosci 2012; 35(10): 1622-1629.

46. Long J, Federico P, Perez MA. A novel cortical target to enhance hand motor output in humans with spinal cord injury. Brain 2017; 140: 1619-1632.

47. Maccabee PJ, Amassian VE, Eberle LP, Cracco RQ. Magnetic coil stimulation of straight and bent amphibian and mammalian peripheral nerve in vitro: locus of excitation. J Physiol 1993; 460: 201-19.

48. Macklin RA, Bae J, Orell M, Anderson KD, Ellaway PH, Perez MA. Time-dependent discrepancies between assessments of sensory function after incomplete cervical spinal cord injury. J Neurotrauma 2017; 34: 1778-1786.

49. Maier MA, Olivier E, Baker SN, Kirkwood PA, Morris T, Lemon RN. Direct and indirect corticospinal control of arm and hand motoneurons in the squirrel monkey (Saimiri sciureus). J Neurophysiol 1997; 78: 721-733.

50. Markram H, Lübke J, Frotscher M, Sakmann B. Regulation of synaptic efficacy by coincidence of postsynaptic APs and EPSPs. Science. $1997 ; 275$ : 213-5.

51. Min YS, Chang Y, Park JW, et al. Change of brain functional connectivity in patients with spinal cord injury: graph theory based approach. Annals of Rehab Med 2015; 39: 374-383.

52. Norton JA, Bennett DJ, Knash ME, Murray KC, Gorassini MA. Changes in sensory-evoked synaptic activation of motoneurons after spinal cord injury in man. Brain 2008; 131: 1478-1491.

53. Ozdemir R, Perez MA. Afferent input and sensory function after human spinal cord injury. J Neurophysiol 2017. https://doi.org/10. 1152/jn.00354.2017.

54. Patton HD, Amassian VE. Single and multiple-unit analysis of cortical stage of pyramidal tract activation. J Neurophysiol 1954; 17 : 345-363.

55. Roy FD, Yang JF, Gorassini MA. Afferent regulation of leg motor cortex excitability after incomplete spinal cord injury. J Neurophysiol 2010; 103: 2222-2233.

56. Sakai K, Ugawa Y, Terao Y, Hanajima R, Furubayashi T, Kanazawa I. Preferential activation of different I waves by transcranial magnetic stimulation with a figure-of-eight-shaped coil. Exp Brain Res 1997; 113: 24-32.

57. Sherwood AM, Dimitrijevic MR, McKay WB. Evidence of subclinical brain influence in clinically complete spinal cord injury: discomplete SCI. J Neurol Sci 1992; 110: 90-98.

58. Sommer M, Ciocca M, Chieffo R, et al. TMS of primary motor cortex with a biphasic pulse activates two independent sets of excitable neurons. Brain Stimul 2018; 11 :558-565. https://doi.org/10. 1016/j.brs.2018.01.001

59. Squair JW, Bjerkefors A, Inglis JT, Lam T, Carpenter MG. Cortical and vestibular stimulation reveal preserved descending motor pathways in individuals with motorcomplete spinal cord injury. J Rehabil Med 2016; 48: 589-596.

60. Stefan K, Kunesch E, Cohen LG, Benecke R, Classen J. Induction of plasticity in the human motor cortex by paired associative stimulation. Brain 2000; 123: 572-84.

61. Stefan K, Kunesch E, Benecke R, Cohen LG, Classen J. Mechanisms of enhancement of human motor cortex excitability induced by interventional paired associative stimulation. J Physiol 2002; 543: 699-708.

62. Taccola G, Sayenko D, Gad P, Gerasimenko Y, Edgerton V. And yet it moves: recovery of volitional control after spinal cord injury. Prog Neurobiol 2017.

63. Taylor JL, Gandevia SC. Noninvasive stimulation of the human corticospinal tract. J Appl Physiol 2004; 96: 1496-1503.

64. Taylor JL, Martin PG. Voluntary motor output is altered by spiketiming-dependent changes in the human corticospinal pathway. J Neurosci 2009; 29: 11708-11716. 
65. Tazoe T, Perez MA. Effects of repetitive transcranial magnetic stimulation on recovery of function after spinal cord injury. Arch Phys Med Rehabil 2015; 96: S145-S155.

66. Thickbroom GW. A model of the contribution of late I-waves to alpha-motoneuronal activation: implications for paired-pulse TMS. Brain Stimul 2011; 4: 77-83.

67. Tokimura H, Ridding M, Tokimura Y, Amassian V, Rothwell JC. Short latency facilitation between pairs of threshold magnetic stimuli applied to human motor cortex. Electroencephalogr Clin Neurophysiol/Electromyogr Mot Control 1996; 101: 263-272.

68. Urbin M, Ozdemir RA, Tazoe T, Perez MA. Spike-timingdependent plasticity in lower-limb motoneurons after human spinal cord injury. J Neurophysiol 2017; 118: 2171-2180.
69. Volz LJ, Hamada M, Rothwell JC, Grefkes C. What makes the muscle twich: motor system connectivity and TMS-induced activity. Cereb Cortex 2014; 25: 2346-2353.

70. Werhahn KJ, Fong JK, Meyer BU, et al. The effect of magnetic coil orientation on the latency of surface EMG and single motor unit responses in the first dorsal interosseous muscle. Electroencephalogr Clin Neurophysiol 1994; 93: 138-146.

71. Wolters A, Sandbrink F, Schlottmann A, et al. A temporally asymmetric Hebbian rule governing plasticity in the human motor cortex. J Neurophysiol 2003; 89: 2339-2345.

72. Ziemann U, Tergau F, Wassermann EM, Wischer S, Hildebrandt J, Paulus W. Demonstration of facilitatory I wave interaction in the human motor cortex by paired transcranial magnetic stimulation. J Physiol 1998; 511: 181-190. 